REVI EWS

\title{
Psychological distress among targets for lateral violence: A conceptual framework
}

\author{
Ericka Sanner-Stiehr, Peggy Ward-Smith \\ University of Missouri, Kansas City, USA
}

Correspondence: Peggy Ward-Smith. Address: School of Nursing, University of Missouri, Kansas City, Kansas City, MO. 2646 Charlotte Street, Kansas City, MO 64108, USA. Email: wardsmithp@umkc.edu.

Received: September 11, 2012

Accepted: October 7, 2012

Online Published: January 4, 2013

DOI : $10.5430 /$ jnep.v3n6p84

URL: http://dx.doi.org/10.5430/jnep.v3n6p84

\section{Abstract}

Incidences of lateral violence frequently occur within the profession of nursing. The Nurse as Wounded Healer theoretical framework provides a blueprint which nurses in staff development can use to identify and guide individuals who have endured lateral violence. This intervention may decrease incidences of lateral violence and improve staff nurse retention.

\section{Key words}

Psychological distress, Lateral violence, Conceptual framework, Nurse retention

\section{I ntroduction}

Lateral violence (LV) as a form of workplace bullying has been defined as both verbal and non-verbal behavior, occurs between and among nurses, when one or more intends to devalue, intimidate, undermine, marginalize, or persecute a target individual ${ }^{[1,2]}$. Lateral violence is defined as behaviors which occur repeatedly over a period of time, creating a pattern, as opposed an isolated incident ${ }^{[3-5]}$. Persistent criticism, offensive comments, shouting, or other outbursts of anger are direct behaviors, and thus easily identifiable as LV. Behaviors such as assigning unmanageable or unfair work assignments, gossiping, withholding information, infighting (clique formation), scape-goating (blaming), and a failure to respect privacy and confidences are less direct forms of $\mathrm{LV}^{[6,7]}$ and therefore may be more difficult to identify than direct forms. According to Bartholomew ${ }^{[8]}$, nonverbal covert behavior has the biggest, and most negative, impact. The extent of hostile work environments in healthcare settings has been reported to be as high as $72 \%$ among nurses in critical care areas ${ }^{[3]}$. Hostile work environments are viewed as emotionally, spiritually and psychologically toxic ${ }^{[6,7]}$,

Interventions aimed to sanction the behavior include the necessity of reporting and identifying the perpetrator. This places the perpetrator in a defensive position, which may result in retaliation, thus escalating the situation ${ }^{[9]}$. Effective interventions aimed toward psychological recovery for victims of LV have yet to be identified. The authors assert that the elusive nature of the many nonverbal forms of LV combined with the lack of effective interventions necessitates a more focused conceptual framework. Such a framework can facilitate development of appropriate interventions. In this article, the authors posit that combining the Transactional Model and Coping ${ }^{[10]}$ and Nurse as Wounded Healer ${ }^{[11]}$, theoretical framework, one can identify when and how to provide an appropriate, sensitive, and respectful intervention to assist victims. 


\section{Framework development}

Research thus far has addressed LV from administrative, policy, or reporting procedural perspectives, there is a paucity of research focused on the development of interventions which may be initiated by the victim. Walker and Avant ${ }^{[12]}$ recommended that a concept analysis be performed when there is little research available, or when the internal structures of a concept need to be clearly described and defined. Using this method to develop a framework, specific to describing LV and including the psychological distress as an outcome, begins with identifying salient concepts and positing the relationships between the concepts.

Guided by the steps of concept analysis ${ }^{[12]}$, the concepts under investigation are LV and psychological distress. The aim of the framework is to describe the process (es) that may occur in the presence of LV and identify steps that can decrease or alleviate psychological distress as an outcome. The identified attributes, relationships between and among the constructs, and consequences are depicted in Figure 1 and described elsewhere in this article.

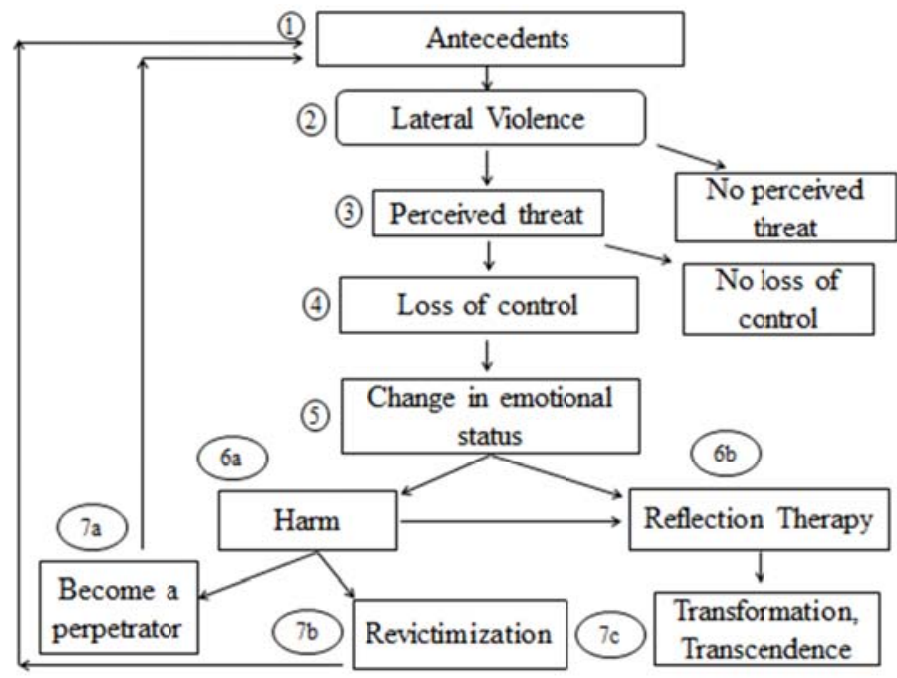

Figure 1. Schematic of proposed conceptual framework with combined components of Transactional Model of Stress and Coping ${ }^{[5]}$ and Nurse as Wounded Healer ${ }^{[6]}$

\subsection{Psychological distress}

Psychological distress, as an outcome or consequence of LV, may take many forms. Within the literature, this distress has been characterized as a discomfort, or a negative shift away from a target's baseline comfort ${ }^{[13,14]}$. In addition to discomfort, Ridner ${ }^{[14]}$ characterizes psychological distress as the perception of an inability to cope effectively, a change in emotional status, communication of discomfort, and harm. Quine ${ }^{[15]}$ posits that targets of LV perceive a loss of control over their situation or lack the resources to effectively respond to incidences of LV. The perception of a loss of control over a stressor, in this case, LV, has been identified by Hutchinson and colleagues ${ }^{[16]}$ as a critical attribute for psychological distress. When targets of LV perceive having the personal and/or professional resources for managing the situation, the shift from baseline comfort and changes in emotional status are temporary. When targets of LV lack effective coping resources, comfort and emotional status changes are sustained, resulting in harm.

Jackson and colleagues ${ }^{[17]}$ suggest report that underreporting of LV may occur due to perceived lack of administrative support. This lack of support results in long-lasting psychological harm, increased absenteeism ${ }^{[18]}$, job attrition ${ }^{[19]}$, and exiting the profession ${ }^{[20]}$. The Institute of Medicine ${ }^{[21]}$ has linked LV to a deterioration of patient care due to being distracted or intimidated. The Joint Commission ${ }^{[22]}$ issued a sentinel event warning which named disruptive behaviors in the workplace as a source of clinical errors and negative patient outcomes. 
It is important to note that the discomfort associated with LV may be manifested subtly and seem unrelated to LV. Staff turnover and leaving the nursing profession have been linked to LV in the literature as symptoms of ineffective coping by targets ${ }^{[23]}$. What appears to be social withdrawal may be socially forced isolation as a result of infighting or simply a passive effort to avoid being further targeted. Yet others may react to LV with aggression, fighting back at the perpetrator or lashing out at others as a viable defense mechanism ${ }^{[24-26]}$. Research results of Woelfle and colleagues ${ }^{[27]}$ determined that when LV occurs in critical care areas, negative patient outcomes are more likely to occur. Early identification of consequences of LV, such as changes in emotional states, allows assessment to occur, which provides timely intervention. Early, appropriate intervention will not only prevent or minimize the psychological stress associated with LV, but it may prevent the target from leaving the employer, or profession.

Multiple references have correlated LV to anxiety, depression, and decreased self- esteem and confidence ${ }^{[7,25,28,29]}$. Hershcovis and colleagues ${ }^{[18]}$ state that once these characteristics are present, the LV target actually increases the possibility for re-victimization. Nurses who are perceived present as depressed, anxious, and unsure of themselves may be identified as weak and unable to defend themselves, making them easy targets for perpetrators of LV. When re-victimization occurs these characteristics become reinforced. This results in LV becoming a cyclical event for both the target and perpetrator. LV has also been described as a learned behavior ${ }^{[24]}$. Whether LV is personally experienced or simply witnessed, these experiences allow the perpetrators and victims to learn these behaviors. Furthermore, if LV regularly occurs without consequence nurses learn that these behaviors are acceptable.

\subsection{Nurse as wounded healer}

The theory of Nurse as Wounded Healer ${ }^{[11]}(\mathrm{NWH})$ provides a framework for describing the psychological distress associated with LV and identifying where intervention may be appropriate to break the cycle. Within this theory, Conti-O'Hare describes the negative psychological outcomes of previously sustained traumas. NWH was conceptualized to explain the difficulties addictions nurses face in caring for their patients if they, themselves, have had close relationships with addicts. Within this theory, psychological trauma can be sustained when issues stemming from these relationships are not addressed and resolved. Conti-O'Hare does suggest that engaging in steps of reflection can help targets assign and understand meaning to their past traumas and eventually transform those traumas into something less harmful. Once the traumas of past experiences are transformed, they can be transcended, which allows the nurse to care for addictions patients, using his/her therapeutic self. The nurse is also then able to shed the deleterious worldview that the past traumas created.

Using the NWH theoretical framework ${ }^{[11]}$, healing is holistic and identifies harmony between the individual and all facets of life as the outcome. After instances of trauma, healing does not erase the memory of the negative event; rather the meaning of the event is transformed, making it non-threatening. Once the negative feelings are neutralized, the individual can transcend them and move forward in life with a more positive outlook.

Traumas, in the presence of lateral violence, are psychological and thus highly subjective. Conti-O’Hare ${ }^{[11]}$ suggests that an individual may not recognize harm as a result of a traumatic event. Thus, discussing past traumatic events may be confusing or painful, if they have not been appropriately recognized and resolved. Recalling past traumas may be met with resistance, as individuals may resist "opening the wound even though relief can be anticipated (p.55)" "11]. Nurses, as a collective profession, tend to strive to live up to an expected image of perfection, able to care for others without absorbing the negative impacts. Attempting to conform to such an image may prevent nurses from even acknowledging the possibility that trauma could happen to them. Despite numerous data demonstrating the frequency of LV and its enculturation within the profession of nursing, there is a scarcity of theoretical frameworks useful to identify, guide, or intervene when LV occurs. Addressing LV from the viewpoint of helping victims has the potential to improve nurse retention both to the job and the profession as well as improve patient outcomes. 
Within the NWH theory there are five steps which are delineated in the QUEST model. The steps within QUEST are identified as Question, Uncover, Experience, Search for Meaning, and Transform and Transcend. QUEST describes the five steps of reflection and provides a blueprint for healing ${ }^{[11]}$. Reflective practice occurs at each step, allowing the individual to gain insight into both the trauma and themselves. Once the trauma has been accepted (Question), the individual must understand its personal meaning and how it has impacted his or her life (Uncover). According to Conti-O’Hare, Uncovering may be the most difficult step in the process because it includes acknowledging one's own behaviors which may have been undesirable. For example, when victims of LV become perpetrators, they must take responsibility for causing psychological distress in others. On the other hand, if they have been re-victimized and developed low self-esteem or depression, they may view themselves as weak for not having defended themselves. It is not possible to guarantee that any intervention will be implemented. However, this framework identifies possible proactive junctures which may be amendable to intervention, initiated by the victim.

Next, the individual must go beyond acknowledging the trauma to processing and experiencing it on an emotional level (Experience). Searching for Meaning allows the trauma to be understood in ways that cannot be articulated in words but rather an assimilation of feelings toward the event. Victims of LV may need to come to terms with their roles in the events, their actions, or their emotional changes. They may have guilt over harming others in an attempt at self-defense or feelings of inadequacy related to their inability to defend themselves. Reconciling these feelings may be an emotional strenuous process. However, once the underlying meaning of the trauma is fully comprehended, it is possible to Transform the past event, neutralizing its negative effects, and transcend it. If an individual is able to transform the trauma by viewing it with a new perspective and self-awareness, the suffering can be transcended and a more positive worldview of the future can be formulated. Those who are able to Transform and Transcend past traumas of LV are able to respond to future transgressions more effectively, interrupting the cycle. Successful Transcendence also allows the individual to reach out therapeutically to others who have experienced similar traumas.

\subsection{Transactional model of stress and coping}

LV is a consistently cited source of stress in the nursing workplace. Nurses' personal coping abilities and past experiences determine their perceptions of $\mathrm{LV}$ and their reactions to it. If targets perceive themselves to possess effective coping, they will view LV as less of a threat and respond differently than those who feel unequipped. Lazarus and Folkman's ${ }^{[10]}$ transactional model for stress and coping ${ }^{[5]}$ describes these initial steps of responding to stressful events. There are two steps in this model: primary and secondary appraisal. During the primary appraisal stage, the individual begins to seek answers with respect to the meaning of the situation. During this stage, the situation is perceived as irrelevant, good, or stressful - in short, whether it poses a threat. Personal factors, such as prior exposure, may influence the perception and intensity of the event. If the event is determined to be threatening, a secondary appraisal is performed.

During the secondary appraisal, the individual considers what do be done in response to the stressor. The individual's evaluation of their resources and coping ability, is weighed against what is needed to effectively handle the stressor. If the target perceives that he or she is able to manage the situation effectively, then the situation can be addressed proactively. If coping or other resources are not present on secondary appraisal, psychological distress results.

\subsection{Reconceptualization of the theories}

Expanding the transactional nature of stress, as defined by Lazarus and Folkman ${ }^{[10]}$, and including the steps outlined in QUEST, provide a conceptual framework (see Table 1) aimed capable of describing and displaying the steps required to assess and reflect (Step 6b) on the trauma experienced as a result of LV (Step 6a) and transform psychological distress (Steps 5 and 7c). Transformation of the LV traumas also eliminates the need for a negative worldview of nurse-nurse relationships, allowing the target to relinquish target status and interrupting the cycle of LV.

Transformation of psychological distress breaks the cycle of LV by alleviating the need respond to aggression with aggression out of retaliation or self-defense as shown in Step 7a of the proposed model. Personal growth may also result 
from successful reflection following distress. Woelfle and colleagues ${ }^{[27]}$ note that contemplation about the distress and "a positive revision of personal schemas" (p. 123) can result in growth. Within this proposed model, achieving personal growth from psychological distress also means that individuals may be better prepared to deal with LV if they are targeted again. Steps 5 and 6a may be eliminated in subsequent situations. Thus, reflecting upon the trauma of LV (theoretically) both prevents targets from becoming perpetrators and helps them to perceive an ability to manage similar situations in the future.

\section{I mplications for research}

Exploring the relationship between LV and psychological distress requires a framework which specifically examines the progression of events, manifestation of symptomology, and identifies outcomes. Therefore, an appropriate and useful conceptual framework must include the entire cycle from antecedents of LV perpetration to demonstration of symptoms, and finally consequences as antecedents. A primary advantage of using this more specific model may be to develop, implement, and study the effects of any intervention(s).

If reflective therapy, as defined by the QUEST model, is implemented to decrease psychological distress secondary to LV, this model will serve as a predictive tool for outcomes. This framework could then be implemented in studies to determine nurses' outcomes after the therapy, the incidence of becoming a Walking Wounded again in the future, or becoming a future perpetrator of LV. If interventions are successful in reducing psychological distress in targets and breaking the cycle of LV, this model would also be useful in implementing policy changes for institutions to provide therapy for these targeted people.

Challenges include when exploring LV and psychological distress include multiple closely related concepts and sub-concepts, many with similar defining attributes, that are frequently and interchangeably, used in the literature. This ambiguity in terminology is perceived as problematic for research ${ }^{[7]}$. The use of varied terminology with respect to defining LV in the workplace may contribute to underreporting these events. This, coupled with the ineffectiveness of administration to address this phenomenon, perpetuates the LV cycle.

However, in examining the frequency with which the defining attributes of terms used when discussing LV it seems reasonable to posit that there is a link between LV and psychological stress. Thus, an advantage to this framework is its ability to define the concepts of LV and psychological distress and theorize links between the two concepts. First, without this clarification, relationships between concepts must often be inferred in order to create links between attributes of concepts. Secondly, several overlapping terms create confusion for both researchers and readers of research who may have varying presuppositions of a concept's meaning. Therefore, in addition to the aims of examining and ameliorating psychological distress in targets for $\mathrm{LV}$, this conceptual framework adds the contribution of clarifying and synthesizing the existing body of knowledge. There are few interventions and a paucity of theoretical framework which can be used to guide a colleague who has endured LV.

Assisting one in transforming any LV experience assists the person to 'make sense' of the experience and enable them to assist others. According to Bartholomew ${ }^{[7]}$, high-quality relationships, those that are cohesive and reflect solidarity, provide the critical information and psychosocial support needed to provide nursing care. This cohesiveness will not only identify when intervention for LV should occur, but provides a supportive learning clinical environment for new graduates and students. Luparell ${ }^{[30]}$ advocates for the importance of teaching and modeling civility and professional behaviors to nursing students before they enter the nursing workforce. Employment is preferred in healthy work environments, thus not only the nursing profession but out patients will realize positive benefits when LV is identified and intervention occurs. The use of the framework outlined in this article may allow us, as nurses, to begin to develop evidenced-based interventions and outcomes. 
Research, guided by this model will determine its clinical applicability. Application of the model to nurses that have experienced psychological distress as the result of LV while providing an intervention, such as reflective instruction, will provide empirical data capable of objectively assessing the model. Self-assessment before and after the intervention will provide comparative, longitudinal data suitable for quantitative analysis. Revision to any intervention should be based on empirical evidence, which will provide support for this framework, or identify where refinement is needed.

\section{References}

[1] American Nurses Association. Lateral violence and bullying in nursing. 2011. Available from: http://www.nursingworld.org/Mobile/Nursing-Factsheets/lateral-violence-and-bullying-in-nursing.html.

[2] Ohio Nurses Foundation. Breaking the cycle of horizontal violence. ISNA Bulletin. 2011; 37(2): 6-11.

[3] Stanley K, Martin MM, Michael Y, Welton J, Nemeth LS. Examining lateral violence in the nursing workforce. Issues in Mental Health Nursing. 2007; 28:1247-65. PMid:17987480 http://dx.doi.org/10.1080/01612840701651470

[4] Lewis MA. Nurse bullying: Organizational considerations in the maintenance and perpetration of health care bullying cultures. Journal of Nursing Management. 2006; 14:52-8. PMid:16359446 http://dx.doi.org/10.1111/j.1365-2934.2005.00535.x

[5] Einarsen S, Hoel H, Notelaers G. Measuring exposure to bullying and harassment at work: validity, factor structure and psychometric properties of the Negative Acts Questionnaire - Revised. Work \& Stress. 2009; 23:24-44. http://dx.doi.org/10.1080/02678370902815673

[6] Almost J. Conflict within nursing work environments: concept analysis. Journal of Advanced Nursing. 2006; 53:444-53. PMid:16448487 http://dx.doi.org/10.1111/j.1365-2648.2006.03738.x

[7] Embree J, White AH. Concept analysis: nurse-to-nurse lateral violence. Nursing Forum. 2010; 45:166-73. PMid:20690992 http://dx.doi.org/10.1111/j.1744-6198.2010.00185.x

[8] Batholomew K. Ending nurse to nurse hostility. Marblehead MA: HCPro, 2006.

[9] Alspach G. Critical care nurses as co-workers: are our interactions nice or nasty? Critical Care Nurse. 2007; 27:10-14.

[10] Lazarus RS, Folkman S. Stress, appraisal, and coping. New York: Springer. 1974.

[11] Conti-O’Hare M. Nurse as Wounded Healer. Sudbury MA: Jones and Bartlett. 2002.

[12] Walker LO, Avant KC. Strategies for Theory Construction in Nursing (5th ed). 2011. Upper Saddle River NJ, Pearson Education Inc.

[13] Dellasega CA. Bullying among nurses. American Journal of Nursing. 2009; 109: 52-8. PMid:19112267 http://dx.doi.org/10.1097/01.NAJ.0000344039.11651.08

[14] Ridner S. Psychological distress: concept analysis. Journal of Advanced Nursing. 2004; 45: 785-93. http://dx.doi.org/10.1046/j.1365-2648.2003.02938.x

[15] Quine L. Workplace bullying in nurses. Journal of Health Psychology. 2001; 6: 73-84. PMid:22049239 http://dx.doi.org/10.1177/135910530100600106

[16] Hutchinson M, Jackson D, Wilkes L, Vickers M. A new model of bullying in the nursing workplace: organizational characteristics as critical antecedents. Advances in Nursing Science. 2008; 31:E60-71. PMid:18497582

[17] Jackson D, Clare J, Mannix J. Who would want to be a nurse? Violence in the workplace - a factor in recruitment and retention. Journal of Nursing Management. 2002; 10:13-20. PMid:11906596 http://dx.doi.org/10.1046/j.0966-0429.2001.00262.x

[18] Hershcovis MS, Turner N, Barling J, Innes M, LeBlanc M, Arnold K Dupre K et al. Predicting workplace aggression: a meta-analysis. Journal of Applied Psychology. 2007; 92:228-37. PMid:17227164 http://dx.doi.org/10.1037/0021-9010.92.1.228

[19] Longo J, Sherman R. Leveling horizontal violence. Nursing Management. 2007; 38: 34-51. PMid:17491129 http://dx.doi.org/10.1097/01.NUMA.0000262925.77680.e0

[20] American Nurses Association. Lateral violence and bullying in nursing. 2008. Available from: http://www.nursingworld.org/Mobile/Nursing-Factsheets/lateral-violence-and-bullying-in-nursing.html

[21] Institute of Medicine. Keeping Patients Safe: Transforming the Work Environment of Nurses. Washington DC: The National Academies Press; 2004.

[22] The Joint Commission. Sentinel Event Alert Issue 40: Behaviors that undermine a culture of safety. 2008. Available from: http://www.jointcommission.org/sentinel_event_alert_issue_40_behaviors_that_undermine_a_culture_of_safety/

[23] Daiski I. Changing nurses’ dis-empowering relationship patterns. Journal of Advanced Nursing. 2004; 48:43-50. PMid:15347409 http://dx.doi.org/10.1111/j.1365-2648.2004.03167.x

[24] Aquino K, Lamerzt K. A relationship model of workplace victimization: social roles and patterns of victimization in dyadic relationships. Journal of Applied Psychology. 2004; 89: 1023-34. PMid:15584839 http://dx.doi.org/10.1037/0021-9010.89.6.1023 
[25] Hauge LJ, Skogstad A, Einersen S. Individual and situational predictors of workplace bullying: why do perpetrators engage in the bullying of others? Work \& Stress. 2009; 23: 349-58. http://dx.doi.org/10.1080/02678370903395568

[26] Hughes R, Clancy C. Complexity, bullying, and stress: analyzing and mitigating a challenging work environment for nurses. Journal of Nursing Care Quality. 2009; 24: 180-3. PMid:19525756 http://dx.doi.org/10.1097/NCQ.0b013e3181a6350a

[27] Woelfle CY, McCaffrey R. Nurse on nurse. Nursing Forum. 2007; 42: 123-31. PMid:17661804 http://dx.doi.org/10.1111/j.1744-6198.2007.00076.x

[28] Einarsen S, Hoel H, Notelaers G. Measuring exposure to bullying and harassment at work: validity, factor structure and psychometric properties of the Negative Acts Questionnaire - Revised. Work \& Stress. 2009; 23: 24-44. http://dx.doi.org/10.1080/02678370902815673

[29] Glaso L, Matthiasen SB, Nielsen MB, Einarsen S. Do targets of workplace bullying portray a general victim personality profile? Scandinavian Journal of Psychology. 2007; 48: 313-19. PMid:17669221 http://dx.doi.org/10.1111/j.1467-9450.2007.00554.x

[30] Luparrell S. Incivility in nursing: the connection between academia and clinical settings. Critical Care Nurse. 2011; 31:2. PMid:21459868 http://dx.doi.org/10.4037/ccn2011171 